\title{
Study on the relationship of IL-12 and IL-4 levels with macroangiopathy in type 2 diabetes mellitus
}

\author{
Fang Xu, Ziling Li* \\ Department of Endocrinology, The Third Affiliated Hospital of Inner Mongolia Medical University, Baotou, Inner Mongolia \\ Autonomous Region, China
}

Received: July 1, 2016

Accepted: August 13, 2016

Online Published: September 10, 2016

DOI: $10.14725 /$ dcc.v3n3p12

URL: http://dx.doi.org/10.14725/dcc.v3n3p12

\begin{abstract}
Objective: To study the relationship of interleukin-12 (IL-12) and IL-4 levels with macroangiopathy in type 2 diabetes mellitus by comparing the serum IL-12 and IL-4 levels and intima-media thickness (IMT) between the healthy controls and the type 2 diabetic patients with or without macroangiopathy.

Methods: 30 healthy controls and 120 cases with type 2 diabetes mellitus from November 2011 to November 2012 were selected. In accordance with the carotid intima-media thickness, the type 2 diabetes mellitus patients were divided into group A with IMT below $1.0 \mathrm{~mm}$, group B with IMT between $1.0 \mathrm{~mm}$ and $1.2 \mathrm{~mm}$, group C with IMT between $1.2 \mathrm{~mm}$ and 1.4 $\mathrm{mm}$, group D with IMT over $1.4 \mathrm{~mm}$. ELISA was used to measure the levels of serum IL-12 and IL-4 in all groups. And the measurement results of serum IL-12 and IL-4 levels in the groups were compared.

Results: Type 2 diabetes mellitus groups had significantly higher serum IL-12 levels and much lower IL-4 levels than the healthy control group. In the type 2 diabetes mellitus groups, the thicker the IMT was, the higher the incidence of complications became. IL-12 level was positively correlated with IMT while the IL-4 level was negatively correlated with IMT.

Conclusions: IL-12 levels in patients with type 2 diabetes were significantly higher and IL-4 levels were significantly lower than those in the healthy people. In patients with type 2 diabetes, the IL-12 levels increased while the IL-4 levels gradually declined with the advance of macroangiopathy.
\end{abstract}

Key Words: Type 2 diabetes mellitus, Macroangiopathy, Interleukin-12, Interleukin-4

Macroangiopathy is the most common complication of type 2 diabetes mellitus, and atherosclerosis constitutes one of the major pathophysiologic bases of diabetes. Currently, it is recognized that atherosclerosis is a chronic inflammatory reaction, which easily happens to patients with poorly controlled glucose. UK Prospective Diabetes Study (UKPDS) confirmed that good blood glucose control could significantly reduce microvascular complications and prompt a good effect on the prevention and treatment of macroangiopathy, but it failed to reach a great statistical significance $(p=.052)$. It is indicated that other reasons to the exclusion of blood sugar play an important role in the occurrence and the development of diabetic macrovascular complications. Studies have shown that type 2 diabetes is an inflammatory disease, IL-12 and IL-4 as cytokines play an important role in the occurrence and the development of type 2 diabetes and its complications..$^{[1,2]}$

\section{Data and methods}

\subsection{General Information}

120 cases of type 2 diabetes mellitus from November 2011 to November 2012 were selected from Department of En-

\footnotetext{
*Correspondence: Ziling Li; E-mail: dcc61@ @cspress.com; Address: Department of Endocrinology, The Third Affiliated Hospital of Inner Mongolia Medical University, Baotou, Inner Mongolia Autonomous Region, 014010, China.
} 
docrinology of the Third Affiliated Hospital of Inner Mongolia Medical University, whose cases conformed to the diagnostic criteria established by WHO. Type 2 diabetic patients were divided into four groups according to intimamedia thickness (IMT): group A: IMT $(<1.0 \mathrm{~mm})$, including 15 males and 15 females, with an average age of (57.53 $\pm 7.45)$ years; group B: IMT (1.0-1.2 mm), including 14 males and 16 females, with an average age of $(55.23 \pm 6.46)$ years; group C: IMT (1.2-1.4 mm), including 15 males and 15 females, with an average age of $(59.83 \pm 10.14)$ years; group D: IMT (> $1.4 \mathrm{~mm}$ ), including 20 males and $10 \mathrm{fe}-$ males, with an average age of $(59.27 \pm 9.04)$ years. Thirty healthy controls were healthy examinees during the same period, including 16 males and 14 females, with an average age of $(57.01 \pm 5.93)$ years. Gender and age data in each group had no statistical differences and are comparable.

\subsection{Research methods}

The subjects were fasting for more than 8 hours. FPG, $\mathrm{HbA}_{1 c}$, TG, TC, HDL and LDL were measured on the next day of admission. Resting sitting blood pressure, height and weight were measured on the day of blood sampling to calculate BMI. ELISA was applied to the measurement of IL-12 and IL-4. Measurement of carotid IMT: Siemens ACUSON S2000 Color Doppler Ultrasonic Diagnosis Apparatus was used to examine the carotid artery with the probe frequency of 7.5 MHZ and special operation. Subjects were in a supine position with the head tilted to one side and the carotid artery fully exposed. IMT was mea- sured at a distance of $1 \mathrm{~cm}$ proximal to the bifurcation of common carotid artery on both sides of the neck, and then the IMT average value was calculated for analysis in each group.

\subsection{Statistical methods}

All data were statistically analyzed by use of the statistical software. The measurement data were represented by mean \pm standard deviation $(\bar{x} \pm \mathrm{s})$. In the case of normal distribution and homogeneous variance, analysis of variance was used to compare the mean of each indicator among groups. Linear correlation analysis was performed on the correlation between the two variables. Stepwise regression analysis was applied to the interaction among multiple factors. Categorical data rate was compared by use of chi-square test, taking $\alpha=0.05$ (bilateral) as the test level. The difference $p<.05$ was statistically significant.

\section{Results}

\subsection{Comparison of general information in type $2 \mathrm{di}$ - abetes patients}

By comparing age and BMI in each group, the differences were not statistically significant $(p>.05)$, with comparability. The results showed that with the increase of IMT, the level of IL-12 increased gradually and the level of IL-4 decreased gradually (see Table 1).

Table 1: Comparison of general information in type 2 diabetes $(\bar{x} \pm \mathrm{s})$

\begin{tabular}{|c|c|c|c|c|c|c|c|c|c|c|}
\hline & $\begin{array}{l}\text { Average } \\
\text { Age (Years) }\end{array}$ & $\begin{array}{l}\text { BMI } \\
\left(\mathrm{kg} / \mathrm{m}^{2}\right)\end{array}$ & $\begin{array}{l}\mathrm{HbA}_{1 \mathrm{c}} \\
(\%)\end{array}$ & $\begin{array}{l}\text { TG } \\
(\mathrm{mmol} / \mathrm{L})\end{array}$ & $\begin{array}{l}\text { TC } \\
(\mathrm{mmol} / \mathrm{L})\end{array}$ & $\begin{array}{l}\text { LDL } \\
(\mathrm{mmol} / \mathrm{L})\end{array}$ & $\begin{array}{l}\text { HDL } \\
(\mathrm{mmol} / \mathrm{L})\end{array}$ & $\begin{array}{l}\text { IMT } \\
(\mathrm{mm})\end{array}$ & $\begin{array}{l}\text { IL-12 } \\
\text { (pg/mL) } \\
\end{array}$ & $\begin{array}{l}\mathrm{IL}-4 \\
(\mathrm{pg} / \mathrm{mL}) \\
\end{array}$ \\
\hline $\begin{array}{l}\text { Healthy } \\
\text { Group }\end{array}$ & $57.01 \pm 5.93$ & $24.14 \pm 1.88$ & $5.04 \pm 0.39$ & $1.42 \pm 0.23$ & $4.08 \pm 0.45$ & $2.04 \pm 0.50$ & $1.33 \pm 0.33$ & $(0.59 \pm 0.09)^{\#}$ & $(47.06 \pm 11.22)^{\#}$ & $(633.68 \pm 73.53)^{\#}$ \\
\hline Group A & $57.53 \pm 7.45$ & $25.20 \pm 3.21$ & $(9.37 \pm 2.14)^{*}$ & $(2.93 \pm 3.17)^{*}$ & $(5.67 \pm 2.50)^{*}$ & $(2.85 \pm 0.81)^{*}$ & $1.29 \pm 0.26$ & $(0.83 \pm 0.07)^{* \#}$ & $(54.20 \pm 9.66)^{* \#}$ & $(583 . .48 \pm 83.20)^{* \#}$ \\
\hline Group B & $55.23 \pm 6.46$ & $26.34 \pm 3.98$ & $(9.55 \pm 2.46)^{*}$ & $(2.60 \pm 1.44)^{*}$ & $(5.35 \pm 1.29)^{*}$ & $(2.85 \pm 0.88)^{*}$ & $1.23 \pm 0.23$ & $(1.03 \pm 0.05)^{* \#}$ & $(56.30 \pm 7.75)^{* \#}$ & $(552.93 \pm 874.9)^{* \#}$ \\
\hline Group C & $59.83 \pm 10.14$ & $24.51 \pm 3.83$ & $(9.15 \pm 2.77)^{*}$ & $(2.44 \pm 1.75)^{*}$ & $(5.55 \pm 1.28)^{*}$ & $(3.17 \pm 0.89)^{*}$ & $1.29 \pm 0.26$ & $(1.20 \pm 0.06)^{* \#}$ & $(57.36 \pm 8.38)^{*}$ & $(535.90 \pm 97.66)^{* \#}$ \\
\hline
\end{tabular}

2.2 Different IMT macrovascular complications in type 2 diabetes

Type 2 diabetes patients were compared in their macrovascular complications by use of chi-square test. Hypertension results showed that: group A and group B: $\chi^{2}=6.696$, $p=.010$, the difference was statistically significant; group A and group C: $\chi^{2}=4.344, p=.034$, the difference was statistically significant; group A and group D: $\chi^{2}=9.6$, $p=.020$, the difference was statistically significant. The results of coronary atherosclerotic heart disease showed: group A and group B: $\chi^{2}=4.320, p=.040$, the difference was statistically significant; group $A$ and group C: $\chi^{2}=5.455, p=.021$, the difference was statistically significant; group A and group D: $\chi^{2}=10.756, p=.001$, the difference was statistically significant. The results of cerebral ischemic stroke showed: group A and group D: $\chi^{2}=5.455, p=.026$, the difference was statistically significant (see Table 2).

\subsection{Correlation analysis of IL-12, IL-4, $\mathrm{HbA}_{1 c}, \mathrm{TC}$, TG and LDL with IMT}

The results showed that IL-12, $\mathrm{HbA}_{1 c}$ and LDL were positively correlated with IMT, and the difference was of statis- 
tical significance $(p<.01)$; IL-4 was negatively correlated $<.01$, see Table 3$)$.

with IMT, and the difference was statistically significant $(p$

Table 2: Comparison of type 2 diabetes patients in the incidences of different IMT macrovascular complications

\begin{tabular}{|c|c|c|c|c|c|c|}
\hline \multirow{2}{*}{ Group } & \multicolumn{2}{|c|}{ Hypertension } & \multicolumn{2}{|c|}{ Coronary Disease } & \multicolumn{2}{|c|}{ Cerebral Ischemic Stroke } \\
\hline & No. of Cases & Incidence (\%) & No. of Cases & Incidence $(\%)$ & No. of Cases & Incidence (\%) \\
\hline Group A & 9 & 30.00 & 2 & 6.67 & 0 & 0 \\
\hline Group B & 19 & $63.33^{*}$ & 8 & $26.67^{*}$ & 0 & 0 \\
\hline Group C & 17 & $56.67^{*}$ & 9 & $30.00^{*}$ & 0 & 0 \\
\hline Group D & 21 & $70.00^{*}$ & 13 & $43.33^{*}$ & 5 & $16.67^{*}$ \\
\hline
\end{tabular}

Note. ${ }^{*}$ In comparison with Group A, $p<.05$

Table 3: Correlation analysis of IL-12, IL-4, $\mathrm{HbA}_{1 c}$, TC, TG, LDL with IMT

\begin{tabular}{llllllll}
\hline Indicators & & IL-12 & IL-4 & HbA $_{\mathbf{1 c}}$ & TG & TC & LDL \\
\hline \multirow{2}{*}{ IMT } & $r$ & 0.438 & -0.503 & 0.446 & 0.065 & 0.126 & 0.278 \\
& $p$ & .000 & .000 & .000 & .431 & .125 & .001 \\
\hline
\end{tabular}

\subsection{Linear regression analysis of IL-12 and IL-4 with IMT and related}

Multivariate linear regression analysis of IMT with IL-12, IL-4, IMT, age, TG, TC, LDL and HDL was conducted in a manner of gradual collocation. IMT was a dependent variable, IL-12, IL-4, age, TG, TC, LDL and HDL were independent variables, analysis of variance for regression equation showed that: $F=27.461, p=.000$, with statistical significance. Four independent variables (age, LDL, IL-12 and IL-4) were chosen to the equation. The regression coefficient of age was 0.153 with $95 \%$ confidence interval $(0.001$, 0.010 ); the regression coefficient of LDL was 0.173 with $95 \%$ confidence interval $(0.016,0.101)$; the regression coefficient of IL-12 was 0.352 with $95 \%$ confidence interval $(0.006,0.013)$; and the regression coefficient of IL-4 was -0.415 with $95 \%$ confidence interval $(-0.002,-0.001)$. The results showed that IMT had a linear regression relationship with age, LDL, IL-12 and IL-4. It can be seen from regression coefficients that IL-12 has the greatest effect on IMT and IL-4 has the greatest inhibiting effect on IMT.

\section{Discussions}

Different degrees of lymphocytes infiltration occur in each stage of macroangiopathy caused by type 2 diabetes mellitus, and infiltrating cells are mainly $\mathrm{T}$ cells, to be more exact, are CD4+ T cells. Studies suggest that the activation of Th subgroups and the ratio imbalance of Th1/Th2 are involved in the development of type 2 diabetic macroangiopathy. In accordance with different secreted lymphokines, Th cells can be divided into two subgroups of Th1 and Th2. Th1 cells mainly secrete INF- $\gamma$, IL-2, IL-3, TNF- $\alpha$ and so on, and CD4+ T cells mainly rely on IL-12 to differentiate itself into Th1 subgroup to play a role in cellular immunity, and promote inflammatory reactions. Th2 cells mainly secrete IL-4, IL-5, IL-6, IL-9, IL-10, etc. The differentiation mainly depends on IL-4 and plays an important role in enhancing humoral immunity and inhibiting inflammatory reactions.

IL-12 can not only directly affect the proliferation and the differentiation of $\beta$-cells but also the generation of IFN- $\gamma$, and it is closely related to diabetic macroangiopathy. The experimental results showed that with the increase of IMT, the level of IL-12 gradually increased, and the difference was statistically significant $(p<.05)$. The results suggest that IL-12 is closely related to macroangiopathy caused by type 2 diabetes mellitus, and it plays a role in promoting inflammatory reactions in the occurence and the development of type 2 diabetic macroangiopathy. Type 2 diabetic macroangiopathy may be associated with elevated IL-12 levels in patients with type 2 diabetes. IL-12 can significantly promote the proliferation of Th1 cells, destabilize the ratio imbalance of Th1/Th2 cells, upgrade the response of inflammatory reactions, participate in the lipid metabolism disorders and consequently lead to the occurrence of type 2 diabetic macroangiopathy.

IL-4 is generally considered as an anti-inflammatory cytokine. IL-4 and its receptors, expressed by human pancreatic $\beta$-cells, prevent from $\beta$-cell apoptosis by activating 1-phosphatidylinositol 3-kinase and the pathway of JAK/STAT. Zou et al. ${ }^{[3]}$ used ELISA to determine serum IL-4 and TNF- $\alpha$ levels in 54 patients with diabetes ( 15 cases of type 1 and 39 cases of type 2). The results showed that the IL-4 level of serum in diabetic patients was significantly lower than that in the control group $(p<.05)$. The experimental results showed that, with the increase of IMT, the IL-4 level showed a downward trend with a statistical significance $(p<.05)$. Hence, we consider that IL-4 plays a role in inhibiting inflammatory reactions in vivo and atherosclerosis in patients with type 2 diabetes, and has a protective effect during the occurrence and development of type 2 diabetic macroangiopathy. 
Under the normal circumstance, there is a co-adjustment and interaction relationship between $\mathrm{Th} 1$ and $\mathrm{Th} 2$, of which these two cell functions come to maintain a dynamic balance. Once a certain deviation happens to this kind of balance, the body will probably fall into a disease status, which can lead to a variety of diseases. ${ }^{[4-12]}$ Therefore, enhanced inflammatory reactions exist in patients with type 2 diabetes. Further study of IL-12 and IL-4 will be helpful to enrich the theoretical bases of the pathogenesis of type 2 diabetic macroangiopathy and provide new methods and means for

\section{References}

[1] Liu Y, Peng YD. Inflammatory Mechanism and Prevention and Control Strategy of Insulin Resistance. World Clinical Drugs. 2012; 33(3): 190-192.

[2] Wu XY, Tang XL, Gao Lin. Preliminary Investigation on ImmuneInflammation in Type 2 Diabetes. Chinese Journal of Immunology. 2007; 23(10): 944-946.

[3] Zou JM, Yuan BJ, Wu JY, et al. Detection and Analysis of IL-4 and TNF-a in Patients with Diabetes. Chinese Journal of Coal Industry Medicine. 2000; 3(5): 535-536.

[4] Yan W, Chen Q. Brief Analysis on Inflammatory Mechanism of Type 2 Diabetes/Insulin Resistance. Lishizhen Medicine and Materia Medica Research. 2012; 23(9): 2299-2300.

[5] Zhu L, Li ZC. Research Advances on Chinese Medicine of LGI mechanism of Diabetic Vascular Complications. Chinese Journal of Information on Traditional Chinese Medicine. 2012; 19(8): 107109.

[6] Chen D, Sun Z, Chen L. Research Advances on Acupuncturemoxibustion Treatment for Type 2 Diabetes. Journal of Clinical Acupuncture and Moxibustion. 2012; 28(7): 76-78. prevention and treatment. If inflammatory reactions in vivo can be effectively inhibited in the later clinical treatment, it will be helpful to prevent from the occurrence and development of type 2 diabetic macrovascular complications and improve prognosis.

\section{Conflicts of Interest Disclosure}

The authors have no conflicts of interest related to this article.

[7] Sun Y, Wang W. On Syndrome Differentiation of Diabetes from Dampness, Toxin and Stasis. Inner Mongolia Journal of Traditional Chinese Medicine. 2012; 31(14): 16-17.

[8] Li HZ. Research Advances on Pharmacological Mechanism of Milkvetch Root to Diabetic Nephropathy. Chinese Community Doctors. $2012 ; 14(15)$ : 6-7.

[9] Zhu DL. Inflammation and Type 2 Diabetes. Chinese Journal of Diabetes. 2006; 14(1): 73-74.

[10] Watford WT, Moriquchi M, Morinobu A, et al. The biology of IL12: coordinating innate and adaptive immune response. Cytokine Growth Factor Rev. 2003; 14(5): 361-368. https : //doi .org/10. 1016/S1359-6101(03)00043-1

[11] Amemiya K, Meyers JL, Trevino SR, et al. Interleukin-12 induces a Th1-link response to Burkholderia mallei and limited protection in BALB/c mice. Vaccine. 2006; 24(9): 1413-1420. PMid: 16213631. https://doi.org/10.1016/j.vaccine.2005.09.021

[12] Mueller R. Pancreatic expression of interleukin-4 abrogates insulitis and autoimmune diabetes in nonbese diabetic (NOD) mice. $J$ Exp Med. 1996; 184(3): 1093-1099. PMid: 9064326. https: //doi.org/10.1084/jem.184.3.1093 